\title{
Kindlin-1 expression is involved in migration and invasion of pancreatic cancer
}

\author{
PRAWEJ MAHAWITHITWONG $^{1}$, KENOKI OHUCHIDA $^{1,2}$, NAOKI IKENAGA $^{1}$, HAYATO FUJTAA ${ }^{1}$, \\ MING ZHAO $^{1}$, SHINGO KOZONO ${ }^{1}$, KOJI SHINDO ${ }^{3}$, TAKAO OHTSUKA ${ }^{1}$, SHINICHI AISHIMA ${ }^{3}$, \\ KAZUHIRO MIZUMOTO ${ }^{1,4}$ and MASAO TANAKA ${ }^{1}$
}

\begin{abstract}
Departments of ${ }^{1}$ Surgery and Oncology, ${ }^{2}$ Advanced Medical Initiatives and ${ }^{3}$ Anatomical Pathology, Graduate School of Medical Sciences, Kyushu University; ${ }^{4}$ Kyushu University Hospital Cancer Center, Fukuoka 812-8582, Japan
\end{abstract}

Received March 27, 2012; Accepted May 30, 2012

DOI: $10.3892 /$ ijo.2013.1838

\begin{abstract}
Kindlin-1 is a novel focal adhesion protein that belongs to the kindlin family. Expression of kindlin-1 has recently been reported in lung and colon cancers, but there have been no studies on its expression in pancreatic cancer. This study aimed to investigate the expression and function of kindlin-1 in pancreatic cancer. Quantitative RT-PCR of Kindlin-1 mRNA was performed in various pancreatic cancer cell lines as well as normal pancreatic epithelial cells and fibroblasts. Immunohistochemical analysis of kindlin-1 was performed for pancreatic cancer tissues. The effects of kindlin-1 on the proliferation, migration and invasion of pancreatic cancer cells were investigated using an RNA interference technique. Kindlin-1 mRNA was highly expressed in the pancreatic cancer cell lines, but only slightly expressed in normal pancreatic epithelial cells and fibroblasts. The Kindlin-1 protein was heterogeneously expressed in the cytoplasm and membrane of pancreatic cancer cells, while normal ductal epithelial cells and stromal cells showed no expression. In vitro experiments involving knockdown of kindlin-1 in AsPC-1 and KP-2 cells revealed that the migratory and invasive abilities of the cells were significantly decreased $(\mathrm{P}<0.001)$, while the proliferation abilities were not affected. The present findings suggest that kindlin-1 expression is involved in the progression of pancreatic cancer via enhancement of cell migration and invasion.
\end{abstract}

\section{Introduction}

Pancreatic cancer is one of the most highly aggressive malignancies in the world. Data from the GLOBOCAN series in 2008, estimated 277,000 new cases and 266,000 deaths worldwide (1).

Correspondence to: Dr Kenoki Ohuchida or Dr Kazuhiro Mizumoto, Department of Surgery and Oncology, Graduate School of Medical Sciences, Kyushu University, 3-1-1 Maidashi, Fukuoka 812-8582, Japan

E-mail: kenoki@med.kyushu-u.ac.jp

E-mail: mizumoto@med.kyushu-u.ac.jp

Key words: kindlin-1, pancreatic ductal adenocarcinoma, invasion, migration
Despite developments in diagnosis and treatment, the prognosis of pancreatic cancer remains poor. Only $10-20 \%$ of pancreatic cancer patients undergo potentially curative resection with a median survival of 17-23 months (2). Furthermore, pancreatic cancer responds poorly to most chemotherapeutic agents and radiation, resulting in little prolongation of the survival time in individual groups. The overall 5-year survival rate is $5 \%$, and more than $50 \%$ of patients are at an advanced stage at the time of diagnosis (3). This indicates that there is a great need for novel modalities to assist in diagnostic and therapeutic strategies for management of pancreatic cancer patients.

Kindlin-1 is a novel focal adhesion protein that belongs to the kindlin protein family, and is also known as fermitin family member 1 (FERMT1). The kindlin proteins are composed of a four-point-one, ezrin, radixin, moesin (FERM) domain interrupted by a pleckstrin homology $(\mathrm{PH})$ domain. Kindlin-1 was discovered as a mutated gene related to Kindler's syndrome, an inherited skin disease characterized by skin blistering, atrophy, photosensitivity and generalized poikiloderma (4,5). Kindlin-1 is primarily found in epithelial cells such as keratinocytes and intestinal epithelial cells $(4,6,7)$, while kindlin-2 is expressed ubiquitously and kindlin-3 is exclusively detected in hematopoietic cells (8).

Integrins are key molecules for establishing cell-extracellular matrix (ECM) adhesion, and are involved in the mechanism of cancer progression (9). Several recent publications have indicated the importance of kindlins for integrin regulation and cytoskeletal reorganization (10-13). To initiate intracellular signaling ('inside-out' signaling), kindlin directly binds to $\beta$ integrin tails, leading to integrin activation and triggering increased adhesiveness in the extracellular domain (14). Likewise, in 'outside-in' signaling, kindlin-1 interacts with focal adhesion proteins such as migfilin and FAK, and these interactions link integrins and signaling for reorganization of the actin cytoskeleton $(15,16)$. Therefore, kindlin-1 may play a crucial role in cancer progression via this signaling pathway. Currently, kindlin-1 expression has been reported in colorectal, lung (17) and breast (18) cancers with enhanced cell adhesion, proliferation and motility. However, there have been no reports on the expression of kindlin-1 in pancreatic cancer.

The aims of this study were to evaluate whether kindlin- 1 is expressed in pancreatic cancer cells and to determine its biomo- 
lecular functions. We evaluated Kindlin-1 mRNA expression in pancreatic cancer cell lines, a normal ductal epithelial cell line and cancer-associated fibroblasts using quantitative RT-PCR. We then investigated the molecular functions of kindlin-1, such as its effects on cell proliferation, migration and invasion, in in vitro experiments using pancreatic cancer cell lines.

\section{Materials and methods}

Cells and culture conditions. The following 13 pancreatic cancer cell lines were used: AsPC-1, KP-2, KP-3, Panc-1, SUIT-2 (Dr H. Iguchi, National Shikoku Cancer Center, Matsuyama, Japan), MIA PaCa-2 (Japanese Cancer Resource Bank, Tokyo, Japan), Capan-1, Capan-2, CFPAC-1, H48N, Hs766T, SW 1990 (American Type Culture Collection, Manassas, VA, USA) and NOR-P1 (established by Dr N. Sato in our laboratory). Primary cultures of human normal pancreatic epithelial cells were obtained from Cell Systems (Kirkland, WA, USA) and maintained in Cell Systems Corporation (CS-C) medium containing $10 \%$ fetal bovine serum (FBS), according to the supplier's instructions. In addition, primary cultures of cancer-associated fibroblasts (CAF) derived from five patients with invasive pancreatic cancers were established in our laboratory and used in this study. All cells were maintained as previously described (19).

$R N A$ isolation and $q R T-P C R$. Total-RNA was extracted from cultured cells using a High Pure RNA Isolation Kit (Roche Diagnostics, Mannheim, Germany) according to the manufacturer's instructions. The RNA concentrations were measured using a NanoDrop ND-1000 spectrophotometer (NanoDrop Technologies, Wilmington, DE, USA) at 260 and $280 \mathrm{~nm}$ (A260/280). qRT-PCR was performed using a Chromo4 real-time PCR Detection System (Bio-Rad Laboratories, Hercules, CA, USA) for 40 cycles of $15 \mathrm{sec}$ at $95^{\circ} \mathrm{C}$ and $1 \mathrm{~min}$ at $55^{\circ} \mathrm{C}$ with a QuantiTect SYBR-Green Reverse Transcription-PCR Kit (Qiagen, Tokyo, Japan) according to the manufacturer's instructions. We designed specific primer sequences as follows: FERMT1 (forward primer, 5'-ttgggattcaggaagacagg-3'; reverse primer, 5'-ccctgaccagttgggataga-3'), $\beta$-actin (forward primer, 5'-tgagcgcggctacagctt-3'; reverse primer, 5'-tccttaatgtcacgcac gattt-3') and $18 S$ rRNA (forward 5'-gtaacccgttgaacccatt-3'; reverse 5'-ccatccaatcggtagtagcg-3'. We performed BLAST searches to ensure the specificity of these primers. All primers were purchased from Sigma Genosys (Tokyo, Japan). The expression of each mRNA was calculated from a standard curve constructed with total-RNA from SUIT-2 cells and normalized by the expression of $\beta$-actin or $18 S$ rRNA.

Immunohistochemistry for human pancreatic cancer tissues. Pancreatic cancer tissues were obtained from patients who underwent pancreatic resection for pancreatic cancer at our institution. We also obtained normal pancreatic tissue samples from intact pancreas resected for bile duct cancer as control tissues. Serial $3 \mu \mathrm{m}$ sections were prepared from the selected paraffin blocks, deparaffinized in xylene and rehydrated in ethanol. Endogenous peroxidase was blocked by incubation in $3 \%$ hydrogen peroxide in methanol for $30 \mathrm{~min}$. Antigen retrieval was achieved by heating in a microwave in citrate buffer at pH 6.0. A Histofine SAB-PO Kit (Nichirei, Tokyo,
Japan) was used for immunohistochemical labeling. The sections were incubated with a rabbit polyclonal anti-kindlin-1 antibody (Millipore, Billerica, CA, USA; 1:100 dilution) overnight at $4^{\circ} \mathrm{C}$. The sections were then incubated with a biotinylated anti-rabbit immunoglobulin solution for $20 \mathrm{~min}$ followed by a 20 -min incubation with peroxidase-labeled streptavidin. The reaction products were visualized using 3,3'-diaminobenzidine as a chromogen, and the nuclei were counterstained with hematoxylin. The study was approved by the Ethics Committee of Kyushu University and conducted according to the Ethical Guidelines for Human Genome/Gene Research enacted by the Japanese Government and the Helsinki Declaration.

Silencing of kindlin-1 using small interfering RNAs (siRNAs). AsPC-1 and KP-2 cells were transfected with kindlin-1 I (sense, 5'-cauguagauucuggacuaatt-3'; antisense, 5'-uuaguccagaaucuaca ugtt-3') and kindlin-1 II (sense, 5'-gagaugugaccaugagaautt-3'; antisense, 5'-auucucauggucacaucuctt-3') siRNAs (Sigma Genosys) by electroporation using a Nucleofector system (Amaxa Biosystems, Koln, Germany) according to the manufacturer's instructions. To verify the specificity of the knockdown effects, we used a control siRNA (Qiagen). All cells were used in the subsequent experiments at 24-96 h after transfection.

Matrigel invasion and migration assays. The invasive ability of pancreatic cancer cells was measured by the number of cells invading Matrigel-coated transwell chambers. Briefly, $1 \times 10^{5}$ KP-2 or AsPC-1 cells were suspended in $250 \mu \mathrm{l}$ of Dulbecco's modified Eagle's medium containing 10\% FBS and placed in the upper transwell chamber $(8 \mu \mathrm{m}$ pore size; Becton Dickinson, Franklin Lakes, NJ, USA) containing $100 \mu \mathrm{l}$ of reconstituted Matrigel-coated membrane (20 $\mu \mathrm{g} / \mathrm{well})$. The upper chamber was placed in a 24-well culture plate containing $750 \mu 1$ of the above-described medium. After incubation for 48 or $72 \mathrm{~h}$ at $37^{\circ} \mathrm{C}$, the numbers of invaded KP-2 and AsPC-1 cells were counted, respectively.

Cell migration assay was performed in pancreatic cancer cells under the same protocol as well as the invasion assay without using Martigrel-coated membrane. Cells were allowed to migrated and counted for 36 (KP-2) or $72 \mathrm{~h}$ (AsPC-1) after cell seeding into the upper chamber. In both assays and at each time point, the cells that migrated and invaded to the bottom side of the inserted chamber were fixed with $70 \%$ ethanol, stained with hematoxylin and eosin, and counted in 10 random fields at x200 magnification under a light microscope. Each experiment was performed in triplicate and repeated at least three times.

Propidium iodide (PI) assay. The proliferative phenotype of pancreatic cancer cell lines was evaluated by PI assay. Cell numbers were counted by measuring the fluorescence intensity of PI at specified times, as described previously (20). Briefly, pancreatic cancer cells were seeded in 24-well plates (Becton Dickinson Labware, Bedford, MA, USA) at $1 \times 10^{4}$ cells/well. After 24, 48, 72 or $96 \mathrm{~h}, \mathrm{PI}(30 \mu \mathrm{M})$ and digitonin $(600 \mu \mathrm{M})$ were added to each well to ensure that all nuclei were labeled with PI. The fluorescence intensities corresponding to the total cells were measured using a microplate reader (Tecan, Männedorf, Switzerland). 


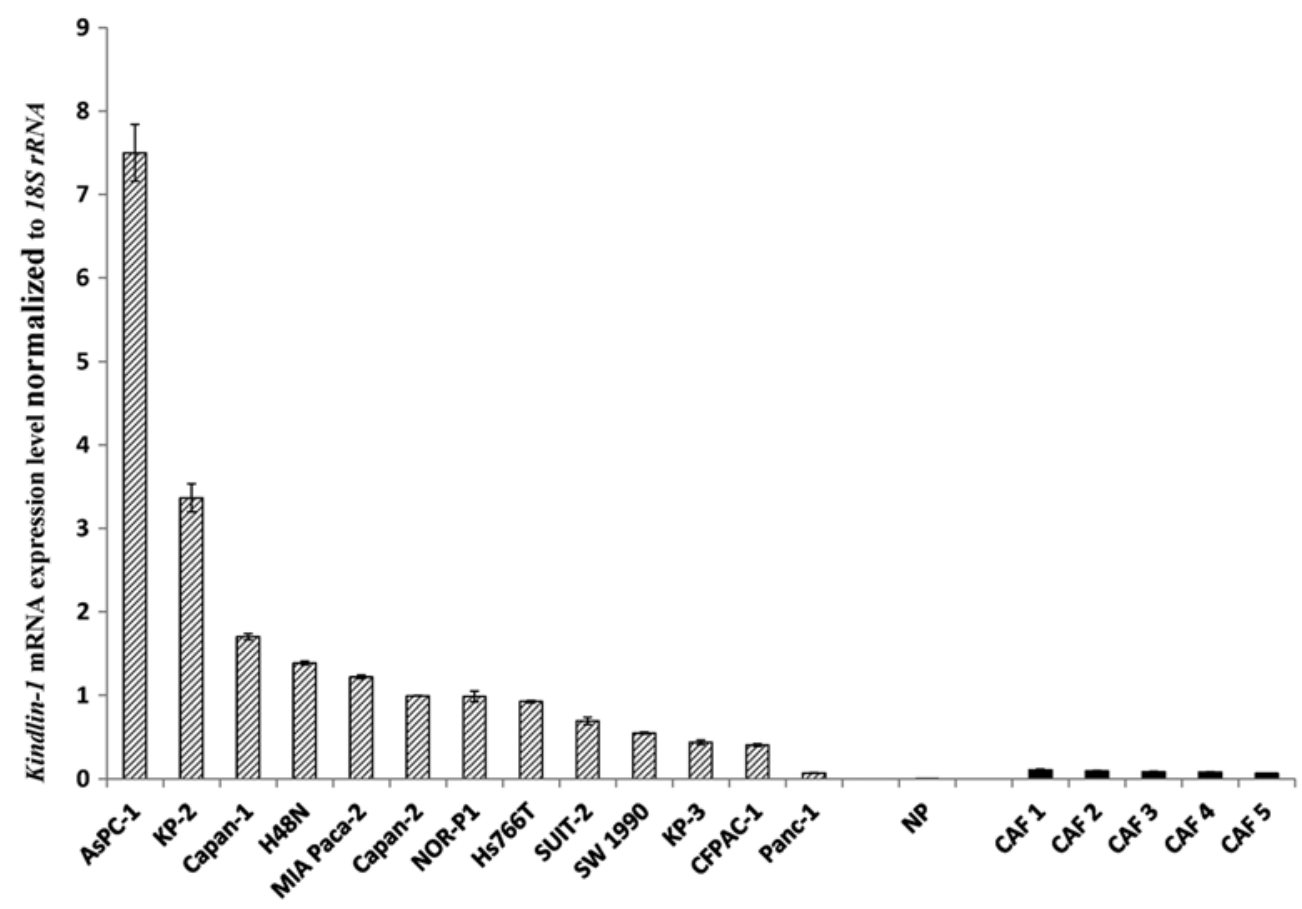

Figure 1. The levels of Kindlin-1 mRNA expression in pancreatic cancer cell lines, human normal pancreatic epithelial cells and cancer-associated fibroblasts were analyzed by qRT-PCR and normalized by the levels of $18 \mathrm{~S} r R N A$ expression. The relative expression levels of Kindlin-1 mRNA in the cancer cell lines are higher than those in normal epithelial cells (NP) and fibroblast cells (CAFs).

Western blotting analysis. All cells were lysed in PRO-PREP ${ }^{\mathrm{TM}}$ (iNtRON Biotechnology, Seongnam, Korea). The cell lysates were fractionated in a Mini-Protean ${ }^{\circledR}$ TGM $^{\mathrm{TM}}$ precast gel (Bio-Rad Laboratories) and transferred to a Trans-Blot ${ }^{\mathbb{B}}$ Turbo $^{\mathrm{TM}}$ Mini PVDF membrane (Bio-Rad Laboratories) using a Trans-Blot Turbo transfer system (Bio-Rad Laboratories). The membrane was incubated overnight at $4{ }^{\circ} \mathrm{C}$ with a rabbit polyclonal anti-kindlin-1 antibody (ab68041; Abcam, Cambridge, MA, USA; 1:1,000 dilution) and then incubated with a horseradish peroxidase-conjugated anti-rabbit $\operatorname{IgG}$ antibody (Cell Signaling, Danvers, MA, USA; 1:5,000 dilution) for $1 \mathrm{~h}$ at room temperature. The bound antibodies were detected using an Amersham $^{\mathrm{TM}}$ ECL $^{\mathrm{TM}}$ Prime Western blotting detection reagent (Amersham Biosciences, Little Chalfont, UK) and visualized with a Molecular Imager (Chemi-Doc XRS System; Bio-Rad Laboratories). The membranes were stripped and probed with an anti- $\beta$-actin antibody (Santa Cruz Biotechnology, Santa Cruz, CA, USA; 1:1,000 dilution) as an internal control.

Statistical analysis. Statistical analyses and graph presentations were carried out using JMP 8 software (SAS Institute, Cary, NC, USA). Values were expressed as the mean $\pm \mathrm{SD}$. Comparisons between two groups were performed using Student's t-test. Statistical significance was defined as $\mathrm{P}<0.05$.

\section{Results}

Kindlin-1 mRNA is highly expressed in pancreatic cancer cell lines compared with normal pancreatic duct cells and fibroblasts. We measured the expression levels of Kindlin-1 mRNA in pancreatic cancer cell lines, human normal pancreatic epithelial cells and primary cultures of cancer-associated fibroblasts by qRT-PCR. There was a wide range of relative Kindlin-1 mRNA expression levels among the pancreatic cancer cell lines, whereas Kindlin-1 mRNA was expressed at very low levels in cancer-associated fibroblasts and normal pancreatic epithelial cells (Fig. 1).

Kindlin-1 is exclusively expressed in pancreatic cancer cells. To elucidate the expression of kindlin-1 in pancreatic tissues, we examined kindlin-1 expression in human samples including pancreatic ductal adenocarcinoma (PDAC) and normal pancreatic tissues adjacent to resected bile duct cancers. Kindlin-1 was exclusively expressed in PDAC, while normal ductal epithelial cells and stromal cells showed no expression (Fig. 2).

Efficacy of siRNAs targeting Kindlin-1. We used AsPC-1 and KP-2 cells, which showed high Kindlin-1 mRNA expression levels, to investigate the biomolecular functions of kindlin-1 in pancreatic cancer cell lines. We inhibited the expression of kindlin-1 using two different siRNAs. At $24 \mathrm{~h}$ (day 1) after transfection with the control siRNA or kindlin-1 siRNAs, the pancreatic cancer cells transfected with the kindlin-1 siRNAs showed lower levels of Kindlin-1 mRNA expression than those transfected with the control siRNA (Fig. 3). Consistently, immunoblot analyses revealed that kindlin-1 protein was also decreased in kindlin-1-knockdown cells at $48 \mathrm{~h}$ after transfection, suggesting good efficacy of both siRNAs for use in the following experiments.

Kindlin-1 has no impact on the proliferation of pancreatic cancer cells. AsPC-1 and KP-2 cells were transfected with the kindlin-1 siRNAs and cultured for $24 \mathrm{~h}$. The transfected cells were then resuspended and seeded in 24-well plates to inves- 

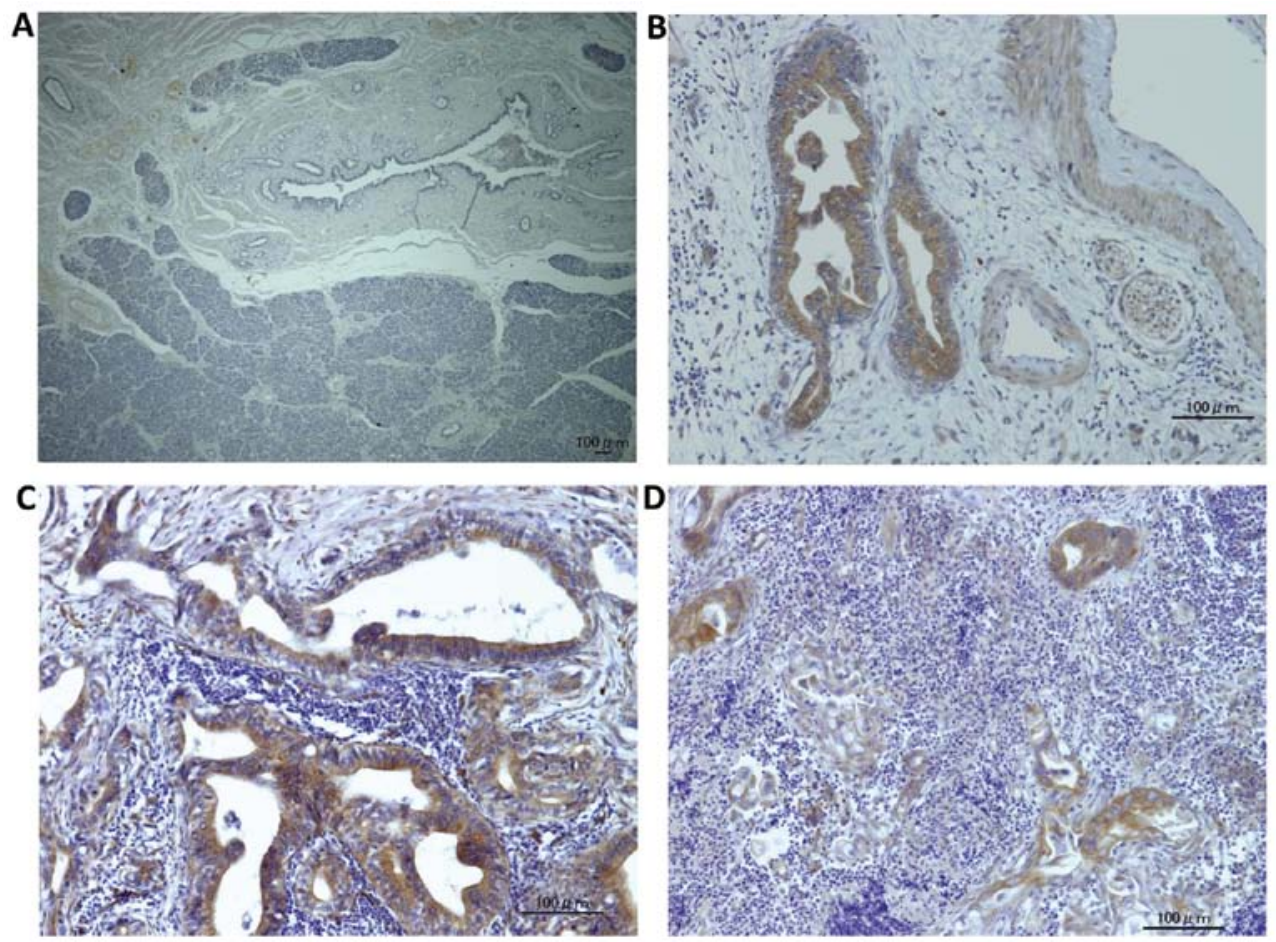

Figure 2. Immunohistochemical analysis of kindlin-1 in human PDAC specimens. (A) Normal pancreas specimens show no kindlin-1 expression in pancreatic ductal epithelial cells and the surrounding stroma. (B-D) In PDAC tissues, immunopositive cells are detected in pancreatic ductal adenocarcinoma cells in various tumor grades, comprising (B) well differentiated, (C) well to moderately differentiated and (D) poorly differentiated tumors. Original magnifications, (A) x40; (B-D) x200.

A

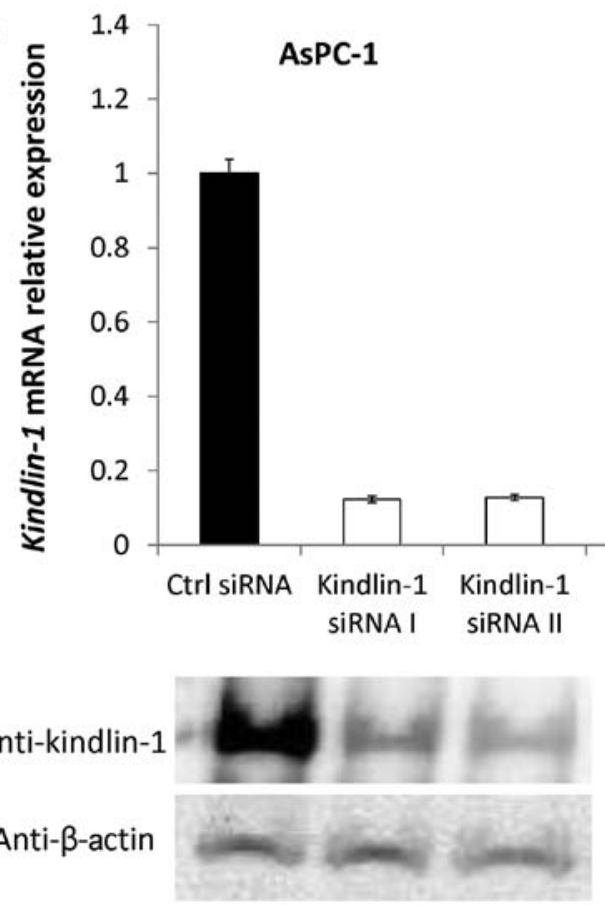

KP-2

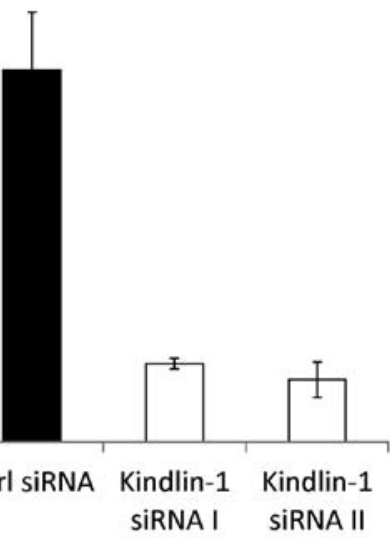

Figure 3. Efficacy of kindlin-1 siRNAs. (A) Transfection of AsPC-1 and KP-2 cells with kindlin-1 siRNA I and kindlin-1 siRNA II leads to reductions in the relative Kindlin-1 mRNA expression levels at $24 \mathrm{~h}$. (B) The kindlin-1 protein levels are also decreased at $48 \mathrm{~h}$ after knockdown with the two different siRNAs in AsPC-1 and KP-2 cells.

tigate the effects of kindlin-1 on proliferation. Increasing cell numbers were observed and counted on the indicated days, and the growth rates of the kindlin-1-deficient cells were similar to those of cells transfected with the control siRNA (Fig. 4). These findings suggested that kindlin-1 had no effect on the proliferation of pancreatic cancer cells. 

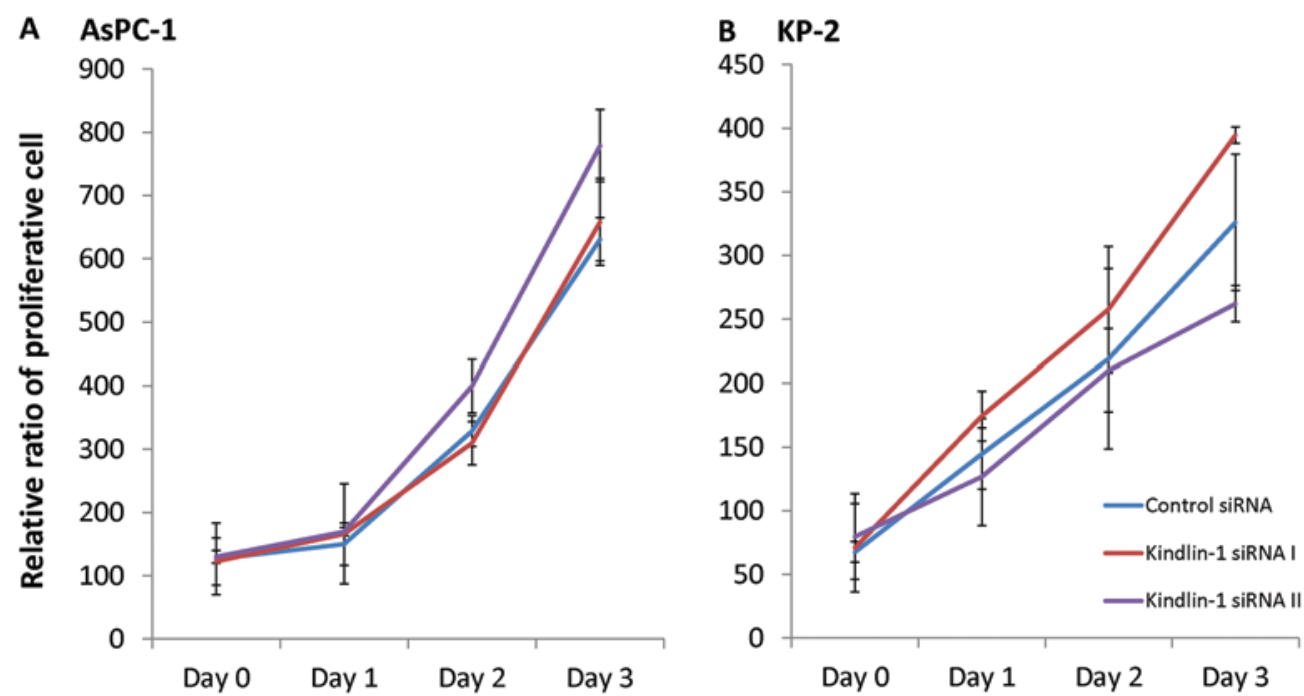

Figure 4. Knockdown of kindlin-1 has no effect on the proliferation of pancreatic cancer cell lines. (A and B) Pancreatic cancer cell lines were transfected with the two different siRNA against kindlin-1 or a control siRNA for $24 \mathrm{~h}$. The proliferative effect of the siRNA-transfected cells was assessed by PI assay every $24 \mathrm{~h}$ for 4 days on (A) AsPC-1 cells and (B) KP-2 cells. No effects on the proliferation of the pancreatic cancer cell lines are observed after knockdown of kindlin-1.

A AsPC-1
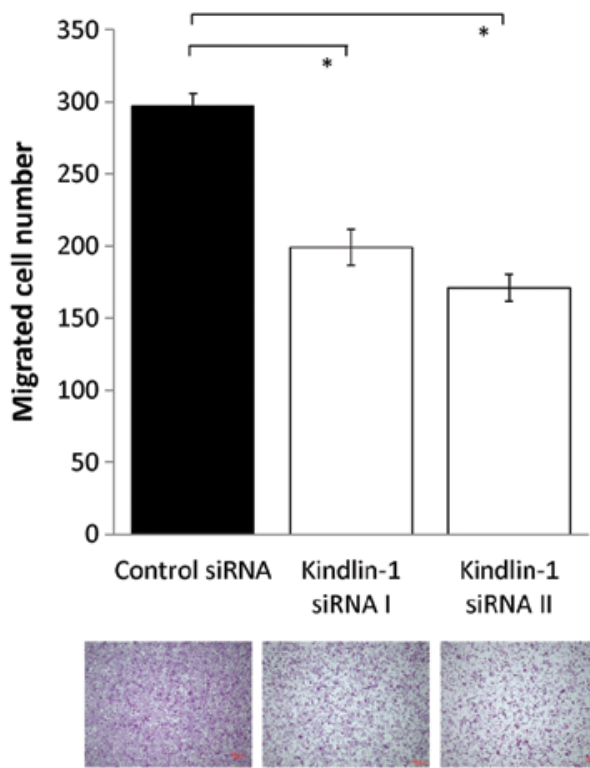
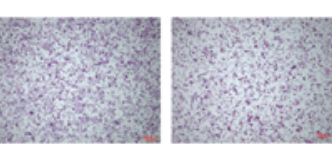

B KP-2
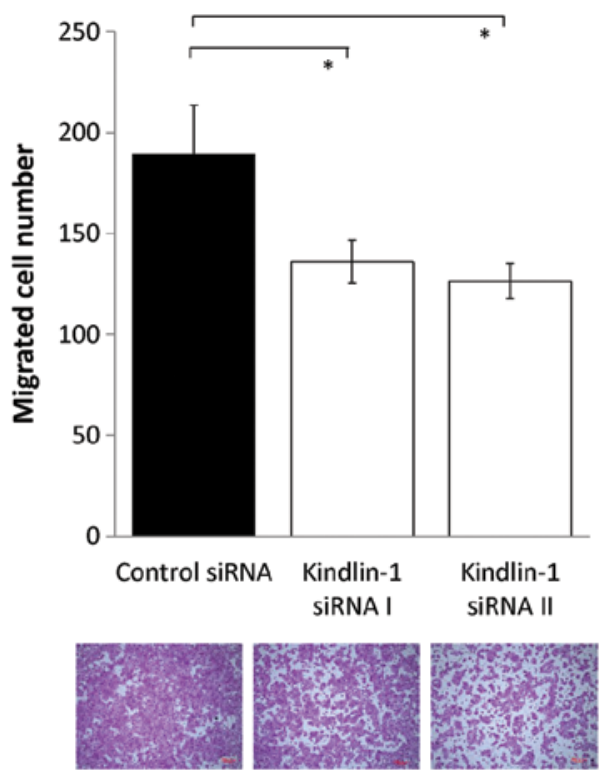

Figure 5. Knockdown of kindlin-1 reduces the migration of cancer cells. (A and B) Pancreatic cancer cell lines were transfected with two different siRNAs against kindlin-1 or a control siRNA for $24 \mathrm{~h}$. The migratory ability of the siRNA-transfected cells was assessed by migration assays after (A) $72 \mathrm{~h}$ for AsPC-1 cells and (B) $36 \mathrm{~h}$ for KP-2 cells. The numbers of migrated cells are shown in the upper panels as means $\pm \mathrm{SD}$ ( $\mathrm{P}<0.001)$ and representative photomicrographs of migration assays are shown in the lower panels (x40 magnification).

Inhibition of kindlin-1 expression decreases the abilities for migration and invasion in pancreatic cancer cells. Next, we investigated the effects of inhibition of kindlin-1 expression on the migratory and invasive abilities of pancreatic cancer cells using a double-chamber assay. After inhibition of kindlin-1 expression, the migratory abilities of AsPC-1 and KP-2 cells were reduced to $60-70 \%(\mathrm{P}<0.001$; Fig. 5) and their invasive abilities were reduced to $30-50 \%$ ( $\mathrm{P}<0.001$; Fig. 6). These findings indicate that kindlin-1 expression is involved in both the migration and invasion of pancreatic cancer cells and that its impact is greater on invasion than on migration.

\section{Discussion}

This report describes a novel study on kindlin-1 expression and its biomolecular functions in pancreatic cancer. There are a few publications regarding kindlin-1 expression and its relationships with carcinomas. In 2003, Weinstein et al (17) first found that Kindlin-1 mRNA was overexpressed in $70 \%$ of colon carcinoma tissues and $60 \%$ of lung carcinoma tissues by qRT-PCR. Papachristou et al (21) further reported the expression of kindlin-1 in leiomyoma and leiomyosarcoma of soft tissue. They found that kindlin-1 immunoreactivity was exclusively 
A AsPC-1

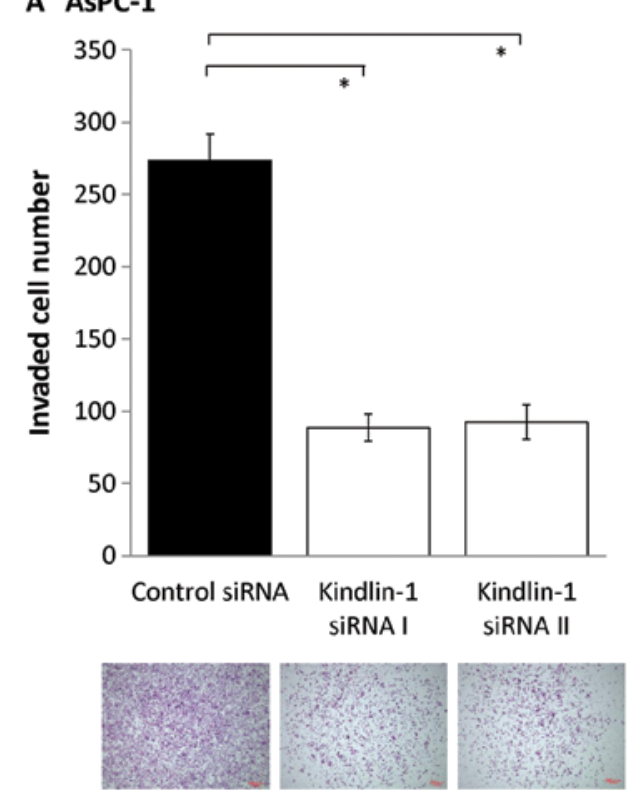

B KP-2

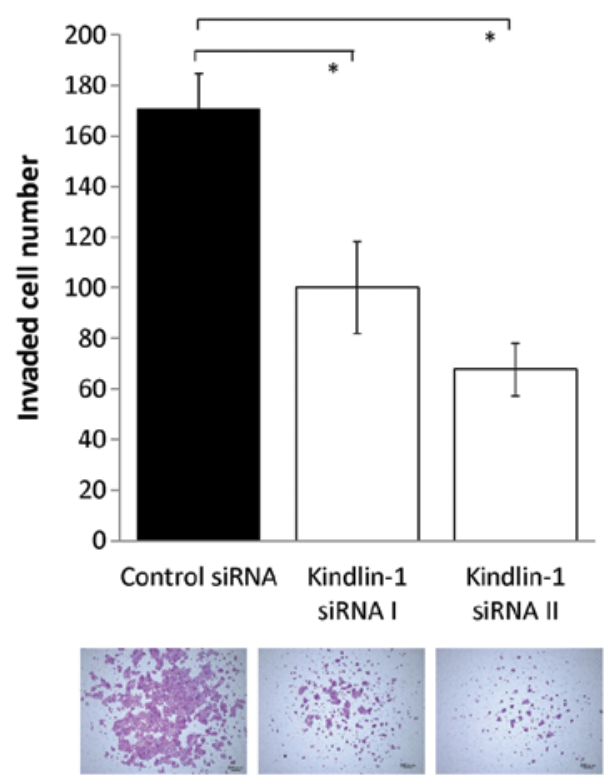

Figure 6. Knockdown of kindlin-1 reduces the invasion of cancer cells. (A and B) Pancreatic cancer cell lines were transfected with the two different siRNA against kindlin-1 or a control siRNA for $24 \mathrm{~h}$. The invasiveness of the siRNA-transfected cells was assessed by Matrigel invasion assays after (A) $72 \mathrm{~h}$ for AsPC-1 cells and (B) $48 \mathrm{~h}$ for KP-2 cells. The numbers of invaded cells are shown in the upper panels as means $\pm \mathrm{SD}\left({ }^{*} \mathrm{P}<0.001\right)$ and representative photomicrographs of invasion assays are shown in the lower panels (x40 magnification).

detected in the cytoplasm of tumor cells, although there were no significant differences between high-grade and low-grade neoplasms and no clinicopathological correlation between kindlin-1 expression and leiomyosarcoma. In breast cancer, both mRNA and protein levels of kindlin-1 have been detected (18). Immunohistochemical analyses further demonstrated strong immunoreactivity in the primary tumors and lung metastases, but not in the adjacent parenchyma or non-lung metastasis sites, suggesting that kindlin-1 is potentially a clinically relevant mediator of lung metastasis of breast cancer and possibly other carcinomas metastasizing to the lung (18). In our study, we found that Kindlin-1 mRNA was highly expressed in various pancreatic cancer cells compared with normal pancreatic epithelial cells and cancer-associated fibroblasts. In addition, immunohistochemical analyses of PDAC and normal pancreatic tissues revealed that kindlin-1 was exclusively expressed in PDAC cells, with no immunoreactivities detected in normal duct cells and stromal cells in pancreatic tissues. Consistent with the previous reports, the present data suggest that expression of kindlin-1 is a promising biomarker for carcinogenesis or progression of PDAC, although investigations of large study groups are required to clarify the clinical implications of kindlin-1.

It is now accepted that the binding between $\beta$-tail integrin and kindlins, in concert with talin, is essential for integrin regulation $(10,22,23)$. In particular, the cytoplasmic tail of $\beta 1$ integrin was shown to be the most important region for kindlin-1 in keratinocytes and intestinal epithelial cells $(6,11)$. Arao et al (24) demonstrated that pancreatic cancer cell lines exhibited high expression of $\beta 1$ integrin, and further showed that the level of constitutive activity of $\beta 1$ integrins was correlated with the invasive ability of pancreatic cancer cell lines. In our study, kindlin-1 expression had effects on both pancreatic cancer cell invasion and migration, but a stronger effect was observed on the invasive ability. However, kindlin-1 had no effect on pancreatic cancer cell proliferation. Although the mechanism remains unclear, these findings may be caused by binding of kindlin-1 to $\beta 1$ integrins, thereby conferring a strong invasive property on the pancreatic cancer cells. However, the partner ligands that bind to kindlin-1, such as talin, may play some hidden roles in the effects on pancreatic cancer cells. Further investigations are needed to elucidate these issues.

It is possible that kindlin-1 may affect pancreatic cancer cell invasion and migration via the TGF- $\beta$ signaling pathway and/or epithelial-mesenchymal transition (EMT), which is the process allowing physiologic and genetic changes of cancer cells from an epithelial phenotype to a mesenchymal phenotype and considered to be an important step in cancer progression and metastasis $(25,26)$. Kloeker et al (7) demonstrated that kindlin-1 expression in human mammary epithelial cell was increased in the presence of TGF- $\beta$ and might also act as a downstream mediator of TGF- $\beta$-initiating EMT. Subsequent data in breast cancer tissues revealed that kindlin-1-expressing cells showed increased cell invasion, migration, clonogenicity and proliferation (18). The study also found that kindlin- 1 initiates TGF- $\beta$-dependent EMT (18). Many studies have clearly shown the role of TGF- $\beta$ signaling and EMT in tumor initiation, progression and metastasis, including pancreatic cancer $(27,28)$. Taken together with our data of in vitro experiments, the decrease in cell invasion and migration after kindlin-1 knockdown in pancreatic cancer cells may involve the TGF- $\beta$ signaling pathway and/or EMT. Elucidation of whether kindlin-1 expression contributes to EMT in pancreatic cancer and other signaling pathways would be beneficial for the development of diagnostic and therapeutic interventions for this disease.

In conclusion, this study provides the first evidence of kindlin-1 expression in pancreatic cancer. Kindlin-1-deficient 
cells showed reduced invasion and migration, but not proliferation. These findings suggest that kindlin-1 is required for pancreatic cancer cell invasion and migration. Further investigations to determine the biomolecular functions of kindlin-1 and its role in pancreatic cancer using both in vivo and clinical studies are required.

\section{Acknowledgements}

This study was supported in part by a Grant-in-Aid from the Ministry of Education, Culture, Sports, Science and Technology of Japan. We are grateful to Nobuhiro Torata, Emiko Manabe and Miyuki Omori (Department of Surgery and Oncology, Kyushu University) for skillful technical assistance.

\section{References}

1. Ferlay J, Shin HR, Bray F, Forman D, Mathers C and Parkin DM: Estimates of worldwide burden of cancer in 2008: GLOBOCAN 2008. Int J Cancer 127: 2893-2917, 2010.

2. Vincent A, Herman J, Schulick R, Hruban RH and Goggins M: Pancreatic cancer. Lancet 378: 607-620, 2011.

3. Siegel R, Ward E, Brawley O and Jemal A: Cancer statistics, 2011: the impact of eliminating socioeconomic and racial disparities on premature cancer deaths. CA Cancer J Clin 61: 212-236, 2011.

4. Siegel DH, Ashton GH, Penagos HG, et al: Loss of kindlin-1, a human homolog of the Caenorhabditis elegans actin-extracellular-matrix linker protein UNC-112, causes Kindler syndrome. Am J Hum Genet 73: 174-187, 2003.

5. Jobard F, Bouadjar B, Caux F, et al: Identification of mutations in a new gene encoding a FERM family protein with a pleckstrin homology domain in Kindler syndrome. Hum Mol Genet 12: $925-935,2003$.

6. Ussar S, Moser M, Widmaier M, et al: Loss of Kindlin-1 causes skin atrophy and lethal neonatal intestinal epithelial dysfunction. PLoS Genet 4: e1000289, 2008.

7. Kloeker S, Major MB, Calderwood DA, Ginsberg MH, Jones DA and Beckerle MC: The Kindler syndrome protein is regulated by transforming growth factor-beta and involved in integrin-mediated adhesion. J Biol Chem 279: 6824-6833, 2004.

8. Ussar S, Wang HV, Linder S, Fassler R and Moser M: The Kindlins: subcellular localization and expression during murine development. Exp Cell Res 312: 3142-3151, 2006.

9. Cabodi S, del Pilar Camacho-Leal M, Di Stefano P and Defilippi P: Integrin signalling adaptors: not only figurants in the cancer story. Nat Rev Cancer 10: 858-870, 2010.

10. Moser M, Legate KR, Zent R and Fassler R: The tail of integrins, talin, and kindlins. Science 324: 895-899, 2009.
11. Harburger DS, Bouaouina $M$ and Calderwood DA: Kindlin-1 and -2 directly bind the $\mathrm{C}$-terminal region of beta integrin cytoplasmic tails and exert integrin-specific activation effects. J Biol Chem 284: 11485-11497, 2009.

12. Plow EF, Qin J and Byzova T: Kindling the flame of integrin activation and function with kindlins. Curr Opin Hematol 16: 323-328, 2009.

13. Goult BT, Bouaouina M, Harburger DS, et al: The structure of the N-terminus of kindlin-1: a domain important for alphaiibbeta3 integrin activation. J Mol Biol 394: 944-956, 2009.

14. Kuijpers TW, van de Vijver E, Weterman MA, et al: LAD-1/variant syndrome is caused by mutations in FERMT3. Blood 113: 4740-4746, 2009.

15. Has C, Herz C, Zimina E, et al: Kindlin-1 is required for RhoGTPase-mediated lamellipodia formation in keratinocytes. Am J Pathol 175: 1442-1452, 2009.

16. Montanez E, Ussar S, Schifferer M, et al: Kindlin-2 controls bidirectional signaling of integrins. Genes Dev 22: 1325-1330, 2008.

17. Weinstein EJ, Bourner M, Head R, Zakeri H, Bauer C and Mazzarella R: URP1: a member of a novel family of PH and FERM domain-containing membrane-associated proteins is significantly over-expressed in lung and colon carcinomas. Biochim Biophys Acta 1637: 207-216, 2003.

18. Sin S, Bonin F, Petit V, et al: Role of the focal adhesion protein kindlin-1 in breast cancer growth and lung metastasis. J Natl Cancer Inst 103: 1323-1337, 2011.

19. Ohuchida K, Mizumoto K, Murakami M, et al: Radiation to stromal fibroblasts increases invasiveness of pancreatic cancer cells through tumor-stromal interactions. Cancer Res 64: 3215-3222, 2004.

20. Zhang L, Mizumoto K, Sato N, et al: Quantitative determination of apoptotic death in cultured human pancreatic cancer cells by propidium iodide and digitonin. Cancer Lett 142: 129-137, 1999.

21. Papachristou DJ, Gkretsi V, Tu Y, et al: Increased cytoplasmic level of migfilin is associated with higher grades of human leiomyosarcoma. Histopathology 51: 499-508, 2007.

22. Harburger DS and Calderwood DA: Integrin signalling at a glance. J Cell Sci 122: 159-163, 2009.

23. Ye F and Petrich BG: Kindlin: helper, co-activator, or booster of talin in integrin activation? Curr Opin Hematol 18: 356-360, 2011.

24. Arao S, Masumoto A and Otsuki M: Betal integrins play an essential role in adhesion and invasion of pancreatic carcinoma cells. Pancreas 20: 129-137, 2000.

25. Thiery JP: Epithelial-mesenchymal transitions in tumour progression. Nat Rev Cancer 2: 442-454, 2002.

26. Iwatsuki M, Mimori K, Yokobori T, et al: Epithelialmesenchymal transition in cancer development and its clinical significance. Cancer Sci 101: 293-299, 2010.

27. Rane SG, Lee JH and Lin HM: Transforming growth factorbeta pathway: role in pancreas development and pancreatic disease. Cytokine Growth Factor Rev 17: 107-119, 2006.

28. Bierie B and Moses HL: TGF-beta and cancer. Cytokine Growth Factor Rev 17: 29-40, 2006. 